\title{
Coding, Ranking and Optimum selection of Nanomaterials
}

\author{
Tanvir Singh ${ }^{1}$ \\ ${ }^{1}$ Department of Mechanical Engineering, DR. B.R. Ambedkar National Institute of Technology, G.T \\ Road Bypass Amritsar, Jalandhar, Punjab-144011, India \\ *Author to whom correspondence should be addressed \\ E-mail: tanvirsingh3@gmail.com, Tel: +918146426766 (Tanvir Singh)
}

\begin{abstract}
The paper presents attribute based characterization of nanomaterials method for computer storage and retrieval as knowledgebase. The knowledgebase permits indepth understanding and comparison between nanomaterials available with the scientists and product developers to satisfy their research and development ( $R \& D$ ) needs. Techniques for order preference by similarity to ideal solution (TOPSIS) is proposed to evaluate nanomaterials in the presence of multiple attributes. The method normalizes attributes to nullify the effect of different units and their values in the range of 0 to 1 . The relative importance of different attributes for different applications is considered. The weight vector is derived using Eigen value formulation. The positive and negative benchmark solutions are derived. Euclidean distance of alternatives from these best and worst solution leads to the development of proximity /goodness/suitability index for ranking. Final decision is taken by decision makers by SWOT analysis and short and long term strategies of the organisation. The methodology is illustrated with the help of an example and step-by-step procedure. Results, discussion and conclusion highlights the importance and practical application.
\end{abstract}

\section{Keywords:-}

Nanomaterial Selection; Pertinent attributes; MADM; TOPSIS; Normalization; Weighted normalization; Coefficient of similarity; Ranking 


\section{Introduction}

Nanomaterial is those materials where the size of the individual building block is less than $100 \mathrm{~nm}$ at least in one dimension. Nanomaterial means any intentionally produced material that has one or more dimensions of the order of $100 \mathrm{~nm}$ or less or is composed of discrete functional parts, either internally or at the surface, many of which have one or more dimensions of the order of $100 \mathrm{~nm}$ or less, including structures, agglomerates or aggregates, which have a size above the order of $100 \mathrm{~nm}$ but retain properties that are characteristic to the nanoscale. Nanomaterial selection is one of the most challenging issues in the design and development of structural elements and it is also critical the success and competitiveness of the manufacturing organisation. The core objective of a material selection procedure is to identify the material selection attributes; such as material cost, product shape, material impact on environment, performance characteristics, availability, fashion, market trends, cultural aspects, aesthetics, recycling, and target group [7] and obtain the most appropriate combination of material selection attributes in conjunction with the feasible requirements. Hence, selection of nanomaterial is a multi attribute decision-making problem. Selection of the appropriate nanomaterial is an integral part of successfully implementation of an engineer's design. The ability to select the most appropriate nanomaterial for a given application is the fundamental challenges faced by the design engineer. A systematic and efficient approach to material selection approach is necessary in order to select the best alternative for a given application [15]. The importance of materials selection in engineering design has been well recognized. The design decision-making regarding selecting appropriate materials is dictated by the specific requirements of an application, often the requirements on materials properties [6]. A multi attribute analysis is a popular tool to select best alternative for given applications. Recent developments in design, the selection of nanomaterial play an important role for engineers. The nanomaterial selection should not be solely based on cost but also depends on different properties of nanomaterial, availability, recycling, production method, disposal method, design life, etc. Selection of nanomaterial depends on number of attributes or factors Thus, great efforts need to be extended to determine attributes that influence nanomaterial selection, using a simple logical approach, to eliminate unsuitable nanomaterial and selection of a proper nanomaterial to be strengthen the existing nanomaterial selection procedure. The selection of an optimal nanomaterial for an engineering design from among various alternatives based on different attributes (i.e. subjective, objective) can be defined within the concept of a multiple attribute decision-making (MADM) problem. So far, it is possible to find various MADM approaches on material selection problem which have already been proposed within previous papers in academic literature [7-13]. There has been rapid increase in the number of nanomaterial and nanomaterial manufacturers. Nanomaterial with vastly different capabilities and specifications are available for a wide range of applications. The selection of the nanomaterial to suit a particular application and production environment, from the large number of nanomaterial available in the market today has become a difficult task. Various considerations such as availability, recycling, production method, disposal method, design life need to be considered before a suitable nanomaterial be selected. The complexity of problem is better appreciated when one realizes that there are over 168 attributes that have to be considered in the selection of nanomaterial for particular application. Moreover, many of them 
are conflicting in nature and have different units, which cannot be unified and compared. The problem of nanomaterial selection for particular application is better appreciated by the Techniques for order preference by similarity to ideal solution (TOPSIS) which is proposed to evaluate nanomaterials in the presence of multiple attributes.

\section{Literature review}

In the past lots of research had been reported for selection of material using classical multi attribute decision-making methods. A multi attribute analysis is a popular tool to select best alternative for given applications and the methods are simple additive weighted (SAW) method, weighted product method (WPM), technique for order preference by similarity to ideal solution (TOPSIS), Vlse Kriterijumska Optimizacija Kompromisno Resenje (VIKOR) method, analytical hierarchy process (AHP), graph theory and matrix representation approach (GTMA), etc. [14-17]. Rao $[16,17]$ presented graph theory and matrix representation approach (GTMA) for the material selection. Shanian and Savadogo [8] presented Elimination and Et Choice Translating Reality (ELECTRE) outranking method for the material selection. Shanian and Savadogo [10] applied TOPSIS method as multiple-criteria decision support analysis for material selection of metallic bipolar plates for polymer electrolyte fuel cell. Manshadi et al. [18] proposed numerical method for the material selection combining non-linear normalization with modified digital logic method. Chan and Tong [19] used grey relational analysis approach (GRA) for multi-criteria selection method. Rao [11] presented improved compromise ranking method for material selection. Rao and Davim [7] described combined multiple attribute decision-making AHP/TOPSIS methodology. Prasenjit et al. [20] used compromise ranking and outranking methods for material selection. Also material selection is carried out using fuzzy decision-making, material design and selection using multi objective decision-making methods [13, 21, 22, 23] Novel method named preference selection index (PSI) method for selection of material for a given application is implemented. By using GTMA a relative importance between attributes are assigned using 8-scales proposed by Chen and Hawang [14] scale proposed by Saaty [24] and the 11-point scale given by Venkatasamy and Agrawal [25]. Rao $[16,17]$ had assigned the relative importance between attribute by considering the 11point scale given by Venkatasamy and Agrawal [25]. Suresh Babu et al. [26] used the TOPSIS method and had considered attributes weight according to an importance and capability of materials. Rao and Davim [7] proposed a logical procedure which is based on a combined technique for order preference by similarity to ideal solution (TOPSIS) and analytical hierarchy process (AHP) method for material selection problem in engineering design. Shanian and Savadogo $[8,9]$ presented a material selection model using a MADM method known as ELECTRE. In another work, Shanian and Savadogo proposed TOPSIS method for material selection of metallic bipolar plates for polymer electrolyte fuel cell. Rao [11] developed material selection methodology using an improved compromise ranking method, also known as VIKOR. Wang and Chang [12] emphasized a fuzzy multiple criteria decision-making approach to help selecting the best suited tool steel material for a specific manufacturing application. Liao [13] took the advantage of a fuzzy multi-criteria decision-making method for material selection. Zadeh [27] presented the concept of fuzzy sets. Bellman and Zadeh [28] presented together with the basic model of fuzzy decision. But the fuzzy sets has limitation, it was extended by vague sets which was presented by Gau and Buehrer [29]. Vague set is generalized form fuzzy sets, vague sets can express and deal with the uncertainty of fuzziness which contain more abundant information. TOPSIS and VIKOR are compromise solutions which are closest to the ideal scheme (Opriumic and Tzeng) [30]. Chu et al. [31] through the VIKOR algorithm can get a compromise solution with a priority . The VIKOR algorithm was proposed by Opricovic [32], which is a multi-attribute decision making method for complex 
system based on ideal point method. The basic view of VIKOR is determining positive-ideal solution and negative-ideal solution in the first place. The positive-ideal solution is the best value of alternatives under assessment criteria, and the negative-ideal solution is the worst value of alternatives under assessment criteria. Ranking by VIKOR may produce different values of criteria weights, criteria weights impact compromise solution. The VIKOR method determines the weight stability intervals, using the methodology presented in Opricovic [33]. Hwang and Yoon [34] presented the technique for order preference by similarity to ideal solution (TOPSIS). TOPSIS takes advantage of positive-ideal solution (PIS) and negative-ideal solution (NIS) of multi-attribute problems to rank the plans sets. In the N-dimensional space, in the plans set A, compare the distances of the alternatives between PIS and NIS, if the plan both near the PIS and far from the NIS is the best plan. Chen (2000) [3] extended the TOPSIS model to the fuzzy environment. Giove [35] presented the interval-valued TOPSIS of multicriteria decision problem. Opricovic and Tzeng [36] pointed out the TOPSIS did not take account of the relative importance of the distance from each point to PIS and NIS.

Even though a good amount of research work was conducted in the past on materials selection, there is a need for a model selection interface to guide practitioners/researchers in matching suitable MADM methods with the material selection decision under different circumstances. To remedy this gap, this paper proposes TOPSIS method selection interface to determine the most appropriate MADM method for different material selection problems. Objective of this research is to generate and maintain exhaustive data of nanomaterial based on their different pertinent attributes. It is useful for better understanding, comparison and analysis and for comparison; ranking and optimum selection of nanomaterial from a large number of nanomaterial available in global market, the multiple attribute decision making problems is solved by "TOPSIS" (Technique for Order Preferences by Similarity to Ideal Solution). It helps the nanomaterial user to save time by providing a tool for selecting the nanomaterial most suited for his operational needs.

\section{Nanomaterial attributes}

Proper identification of nanomaterial attributes is critically important when comparing various Alternative nanomaterials. However, in most cases the user needs to be assisted in identifying the nanomaterial attributes wisely and accordingly as per the considered application. The final product of industry directly depends on the proper choice of the nanomaterials. For this purpose Cause and effect analysis diagram is drawn to identify all the different attributes and other parameters of nanomaterials, which require attention/ situation of designers researchers, industrialist and manufacturer etc. in the subject area under consideration as shown in Figure 1.

\subsection{Identification of Nanomaterial attributes}

The nanomaterial attributes are found out based on its broad area as general parameters, physical parameters, and mechanical, atomic, electrical. Thermal based etc as shown in Table 1.

The above 168 attributes is useful for storage, retrieval, designing, manufacturing, and evaluation, ranking and optimum selection of nanomaterials for R \& D of a nanodevice. Out of 168 identified attributes, there are $\mathbf{7 7}$ attributes in general, $\mathbf{3 5}$ attributes in physical, 19 attributes in mechanical, $\mathbf{6}$ attributes in atomic, $\mathbf{1 0}$ attributes in electrical, $\mathbf{1 4}$ attributes in thermal, and remaining 7 attributes in optical categories.

\subsection{Quantification and measurement of the attributes}


The nanomaterials are expressed in detailed manner with the attributes identified e.g., Young's modulus $107 \mathrm{Gpa}$, Tensile strength $>55 \mathrm{Gpa}$, Aspect ratio 1000 etc. But all attributes are not quantitative, e.g., band structure, morphology, etc. The nanomaterials is rated on the scale of 0-5 for these attributes. A similar approach has to be used for the informative attributes, which just tell information about some attributes of the nanomaterial such as the structure of the nanomaterial or the density of nanomaterial, which is denoted by some number whose numerical value have no significance. It cannot be used for the mathematical treatment since it is just a representation and numeric value has no significance. There are some attributes of which quantification is not readily available and has to be done by some mathematical model or modelling, simulation and analysis Quantification of many of the attributes is not readily available from the manufacturer. But if the manufacturer make it a standard practice to identify, quantify and provide these attributes, it is helpful to him apart from nanomaterial designer, manufacturer, industrialist and user etc

\section{a. Usefulness to the manufacturer}

The quantification and monitoring of the attribute magnitudes help the manufacturer to control them closely so that he fulfils the demand of the user precisely. Moreover, he finds out the market trend by observing the attributes magnitudes. It helps the manufacturer to modify his product to suit the future needs of the nanomaterial user. He uses the data to produce optimum nanomaterials in the minimum possible time. The nanomaterials manufacturer uses these attributes for the SWOT (Strength-Weakness-Opportunity-Threat) analysis of his nanomaterial product. The points to remember for any manufacturer while manufacturing any nanodevice based upon their strength -weakness-opportunity-threat are shown in Figure 2.

\section{b. Usefulness to the designer}

For the designer at conceptual design stage, identification helps to generate various alternative designs, which is developed as modular nanomaterial. Using the modular nanomaterial approach, the optimum nanomaterials according to the market requirements is designed in very little time. He identifies the critical attributes, which directly affects the performance. The designer changes these critical attributes and monitors them to control particular parameters so that the required performance obtained from the nanomaterials. Designer use these attributes for cause and effect analysis, where he find out the effects of manipulating these attributes on the nanomaterials performance.

\section{c. Usefulness to the user}

Identification of the attributes helps the user for the data storage and its retrieval. It generates the computerized data, which is used in different formats for different purposes by different people in the organization. It helps the user to select the best possible nanomaterials for the particular application whenever it is required.

Keeping the short term and long term objectives in mind, comprehensive SWOT analysis by the designer, device manufacturer and $R \& D$ organizations helps in the development of creative and innovative nanodevices.

\subsection{Coding Scheme of Nanomaterial Attributes}


In order to facilitate the selection of pertinent attributes for the application, the attributes are required to be evaluated and coded for range of values. Coding is alphanumeric. The attributes are of two types: quantitative/deterministic and qualitative/fuzzy/subjective. Quantitative attributes determined or calculated using mathematical models or experimentally. Qualitative attributes are subjective in nature and imprecise information is available. It is desirable to evaluate the existence of both types of attributes on one of the several interval scales $0-5$, etc for uniformity. Quantification of many of these attributes is not readily available from the manufacturer. A team of experts from relevant disciplines codify all the attributes related to a particular nanomaterial based on the application that is to be considered. Following examples illustrative the proposed coding scheme for qualitative and quantitative attributes:-

\subsubsection{Quantitative Attributes}

1. Table-2 Coding of specific surface area of nanomaterial. These codes are used to specify the specific surface area of the nanomaterial in the respective shell number 30, since it is allotted to it, as shown in table 6 . Here the nanomaterial under consideration has the specific surface area of $290 \mathrm{~cm}^{2} / \mathrm{g}$, which is given a code 5 as shown in table 7 .

2. Table-3 Coding of tensile strength of nanomaterial. These codes are used to specify the tensile strength of the nanomaterial in the respective shell number 113, since it is allotted to it, as shown in table 6. Here the nanomaterial under consideration has the tensile strength of $400 \mathrm{GPa}$, which is given a code 3 as shown in table 7.

\subsubsection{Qualitative Attributes}

3. Table-4 Coding of magnetic ordering of nanomaterial. These codes are used to specify the magnetic ordering of the nanomaterial in the respective shell number 51, since it is allotted to it, as shown in the table 6. Here the nanomaterial under consideration is diamagnetic in nature, which is given a code $\mathrm{D}$ as shown in table 7.

4. Table-5 Coding of morphology of nanomaterial. These codes are used to specify the morphology of the nanomaterial in the respective shell number 89 , since it is allotted to it, as shown in the table 6 . Here the nanomaterial under consideration having tubular structure, which is given a code $\mathrm{T}$ as shown in table 7 .

The above mentioned attributes tabulated in the form of 168-digit coding scheme for characterization of nanomaterial as shown in Table 6.

\section{Example of coding scheme of standard nanomaterial. " $Q$ Tubes ${ }^{\circledR} 250$ ”}

\section{As shown in Table 7.}

Here, coding scheme for all the 168 attributes are represented for standard nanomaterial under consideration. All these attributes for " $Q$ Tubes ${ }^{\circledR}$ 250" nanomaterial is collected from different research publications and some of them from commercial products and applications.. Table clearly indicates that the information supplied by the manufacturer to the user is meagre and it is required to be more elaborate. Here that most of the cells are having 0 as code in them. The 0 represents that the information relating to the particular cell is not available to the authors. Information is not provided by the manufacturer, but the authors think that if this information is provided it makes the data exhaustive. Moreover, the data storage, retrieval and the selection procedure more precise and accurate. 
Representation of coding scheme for standard nanomaterial "Q Tubes ${ }^{\circledR} \mathbf{2 5 0}$ " can be done for all 168 attributes as shown in Table 8.

The alphabets used in the coding scheme for standard nanomaterial has unique information in itself, like, here material that we are taken for comparison purpose is of black colour represented by letter ' $\mathrm{B}$ ' similarly, it is stable in nature represented by ' $\mathrm{S}$ ', it is insoluble in nature represented by 'IS', for nanomaterial under consideration polymorphism is not done represented by ' $\mathrm{N}$ ', magnetic ordering of nanomaterial is diamagnetic in nature represented by ' $\mathrm{D}$ ' and having a amorphous crystal phase represented by ' $A$ '. It is physical in nature represented by ' $\mathrm{P}$ ', having morphology of tubular structure represented by ' $\mathrm{T}$ '. For this specific suspension is dispersion in water represented by ' $\mathrm{W}$ ', having electrical performance which is conducting in nature represented by ' $\mathrm{C}$ ' Rest of numerical codes are given on the basis of their relative importance highest code i.e. ' 5 ' to highly important attributes and lesser code like ' 4 ', and ' 3 ' to less important attributes and ' 2 ', 'and 1 ' to very less important attributes and ' 0 ' for totally absent attributes. This coding scheme is also used for the visual comparison between two nanomaterials up to certain extent. It allows faster comparison in various formats.

\section{3-Stage Optimum Selection Procedure}

\section{Stage-1 Elimination Search Method}

Though all the attributes have been identified, all of them would not be important while selecting the nanomaterials for particular application. There are few attributes, which have direct effect on the selection procedure. The small number of attributes set-aside as 'pertinent attributes' as necessitated by the particular application and/or the user. The threshold values to these 'pertinent attributes' assigned by obtaining information from the user and the group of experts. Henceforth the selection procedure focuses solely on the pertinent attributes leaving out the rest. On the basis of the threshold values of the pertinent attributes, a shortlist of nanomaterials is obtained. It is achieved by scanning the data for pertinent attributes, one at a time, to eliminate the nanomaterial alternatives, which have one or more pertinent attributes values that fall short of the minimum required (threshold) values. To facilitate that search procedure an identification system has been made for all the nanomaterials in the data.

\section{Stage-2 Evaluation Using TOPSIS Method}

\section{Step-1 Decision matrix}

The first step here is to represent all the information available from the data about these satisfying solutions in the matrix form. Such a matrix is called as decision matrix, D. Each row of the matrix is allocated to one candidate nanomaterial and each column to one attribute under consideration. Therefore an element $\mathrm{d}_{\mathrm{ij}}$ of the decision matrix $\mathrm{D}$, gives the value of $\mathrm{jth}$ attribute in the row (non-normalized) form and units, for the ith nanomaterials. Thus if the number of short-listed nanomaterials is ' $\mathrm{m}$ ' and the number of pertinent attributes is ' $\mathrm{n}$ ' the decision matrix is an $\mathrm{m} x \mathrm{n}$ matrix.

\section{Step-2 Normalized specifications matrix}

The second step is construction of the normalized specification matrix, N, from the decision matrix, D. Normalization is used to bring the data within particular range or scale, and 
moreover it provides the dimensionless magnitudes. The phenomenon is used to calculate the normalized specification matrix. The normalized specification matrix has the magnitudes of all the attributes of the nanomaterial on the common scale of 0 to 1 . It is a sort of value, which indicates the standing of that particular attribute magnitude when compared to the whole range of the magnitudes for all candidate nanomaterials.

An element $\mathrm{n}_{\mathrm{ij}}$ of the normalized matrix $\mathrm{N}$ be calculated as:-

$$
n_{i j}=d_{i j} /\left(\sum_{i=1}^{m} d_{i j}^{2}\right)^{1 / 2}
$$

Where $d_{i j}$ is an element of the decision matrix, D

\section{Step-3 Relative Importance Matrix}

The third step is to obtain information from the user or the group of experts on the relative importance of one attribute with respect to another. The information is sought in terms of a ratio. Information on all such pair-wise comparisons is stored in a matrix called as relative importance matrix, ' $A$ ', which is $\mathrm{n} \times \mathrm{n}$ matrix. Here $\mathrm{a}_{\mathrm{ij}}$ contain the relative importance of $\mathrm{i}^{\text {th }}$ attribute over the $\mathrm{j}^{\mathrm{th}}$ attribute. The symmetric terms of the matrix are reciprocals of each other while the diagonal be unity. The information stored in matrix A is on pair-wise basis. It is to be modified into representation that gives the relative weights of all attributes taken together so that the cumulative sum of the weights is equal to unity.

\section{Step-4 Find out the maximum Eigen value of the relative importance matrix $A$.}

In the fourth step, the Eigen vector method is used, which allows for some inconsistencies in the judgement of relative importance of attributes while making pair-wise comparisons, is used to find out the weights. These inconsistencies arise due to inaccurate human judgments [37]. The Eigen vector method seeks to find weight vector $\mathrm{w}$ from the Eigen value problem associated with the matrix, A, i.e. If

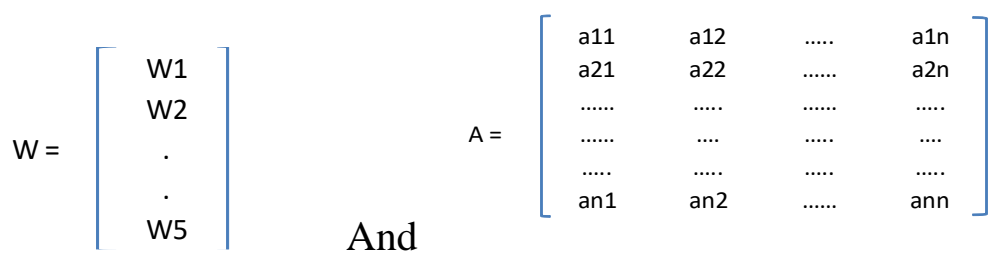

Then the linear transformation $\mathrm{Y}=\mathrm{AW}$

Carries the column vector $\mathrm{W}$ into the column vector $\mathrm{Y}$ by means of the square matrix $\mathrm{A}$. In practice it is often required to find such vectors which transform them into themselves or to a scalar multiple of themselves.

Let $\mathrm{W}$ be such a vector which transforms into $\lambda \mathrm{W}$ by means of the transformation equation (2) Then, $A \mathrm{w}=\lambda \mathrm{w}$ or $\mathrm{AW}-\lambda \mathrm{IW}=0$ or $(\mathrm{A}-\lambda \mathrm{I}) \mathrm{W}=0$ 
Where $\lambda$ the Eigen value of $A$ and $w$ is is the corresponding Eigen vector [38]. For $n \mathrm{x} n$ A there are $n$ Eigen values $\lambda_{i}$, for $i=1, \ldots, n$, and corresponding to $\lambda_{i}$, there are $n$ Eigen vectors $w_{i}$, for $\mathrm{i}=1, \ldots, \mathrm{n}$. vector $\mathrm{w}$ is now found in the following manner:

This Equation is also called Eigen value formulation that provides to find out the weight vector as shown below:-

$(\mathrm{A}-\lambda \mathrm{I}) \mathrm{W}=0$

Where, I is the identity matrix, and $\mathrm{W}$ is the weight vector.

This equation can also be written as

$(\mathrm{A}-\lambda \mathrm{I})=0$

And $\mathrm{W}=0$, Where, $\mathrm{W}$ this gives a trivial solution having no meaning.

Take Eigen weight vector, $W$ corresponding to the largest Eigen value $\lambda_{\max }$, as all the elements of $\lambda_{\max }$ are either positive or negative. [37]. In this way, maximum Eigen value is calculated by using Equation (4).

Step 5 Calculating weights for each attribute using the Eigen vector associated with maximum Eigen value.

In order to find out the weights for each attributes using Eigen vector associated with maximum Eigen value is calculated by using equation (5) as shown below:-

$$
\left(\mathrm{A}-\lambda_{\max } \mathrm{I}\right) \mathrm{W}=0
$$

In this summation of weight vectors $\mathrm{w}_{\mathrm{i}}$ given as

$$
\sum_{i=1}^{n} \mathbf{w}_{i}=1
$$

$$
w_{1}+w_{2}+w_{3}+w_{4}+w_{5}=1
$$

\section{Step-6 weighted normalized specification matrix}

The weights obtained from the relative importance matrix have to be applied to the normalized specifications since all attributes have different importance while selecting the nanomaterials for particular application. The matrix, which combines the relative weights and normalized specification of the candidates, is weighted normalized matrix, ' $V$ '. It gives the true comparable values of the attributes. This is obtained as follows: 


$$
V=\left[\begin{array}{cccc}
w_{1} n_{1,1} & w_{2} n_{1,2} & \cdots & w_{n} n_{1, n} \\
w_{1} n_{2,1} & \ddots & \cdots & \vdots \\
\vdots & \vdots & \ddots & \vdots \\
w_{1} n_{m .1} & w_{2} n_{m .2} & \cdots & w_{n} n_{m, n}
\end{array}\right]=\left[\begin{array}{cccc}
v_{1,1} & v_{1,2} & \cdots & v_{1, n} \\
v_{2,1} & \ddots & \cdots & \vdots \\
\vdots & \vdots & \ddots & \vdots \\
v_{m .1} & v_{m .2} & \cdots & v_{m, n}
\end{array}\right]
$$

\section{Step-7.Generation of +ve and -ve benchmark nanomaterials and separation measures}

The weighted normalized matrix $\mathrm{V}$ is used to obtain the +ve and -ve benchmark nanomaterials, where the both benchmark nanomaterials are hypothetical nanomaterials, which supposed to have best and worst possible attribute magnitudes. The TOPSIS method is based on the concept that the chosen option (optimum) have the shortest distance from the +ve benchmark nanomaterial (best possible nanomaterial) and be farthest from the _ve benchmark nanomaterial (worst possible nanomaterial). The measure ensures that the top ranked nanomaterial is closest to +ve benchmark nanomaterial and farthest from _ve benchmark nanomaterial. Here, calculations are made on separation measures from +ve and _ve benchmark nanomaterial, respectively, as $S_{i}^{*}$ and $S_{i}^{-}$

The separation from the +ve benchmark nanomaterial is given by

$$
S_{i}^{*}=\left[\sum_{j=1}^{n}\left(v_{i j}-v_{1}^{*}\right)^{2}\right]^{1 / 2} \quad(i=1,2, \ldots, m)
$$

And separation from the -ve benchmark nanomaterial is given by

$$
S_{i}^{-}=\left[\sum_{j=1}^{n}\left(v_{i j}-v_{1}^{-}\right)^{2}\right]^{1 / 2} \quad(i=1,2, \ldots, m)
$$

\section{Step-8.Suitability Index}

Then the relative closeness to the +ve benchmark nanomaterial, $\mathrm{C}_{\mathrm{i}}{ }^{*}$, which is a measure of the suitability of the nanomaterials for the chosen application on the basis of attributes considered, is calculated. A nanomaterial with the largest $\mathrm{C}_{\mathrm{i}} *$ is preferable

$$
\mathrm{C}_{\mathrm{i}}^{*}=\mathrm{S}_{\mathrm{i}}^{-} / \mathrm{S}_{\mathrm{i}}^{*}+\mathrm{S}_{\mathrm{i}}^{-} \quad \text { Where } \mathrm{i}=1, \ldots ., \mathrm{m} \text {. }
$$

Ranking of the candidate nanomaterials is done in accordance with the decreasing values of indices $\mathrm{C}_{\mathrm{i}}$ * indicating the most preferred and the least preferred feasible optional solutions. This index may be called as suitability/goodness index.

\subsection{Graphical method based ranking}

Till now have been seen the mathematical representation of the specifications, normalized and weighted normalized specifications. There are many methods to evaluate the nanomaterial using mathematical approach [9]. But trying to explore graphical way to process the available 
data and select the nanomaterials. The graphical representation methods like line graph used for that purpose.

\subsubsection{Line graph representation}

We have specification matrix $\mathrm{D}$, normalized and weighted normalized specification matrices $\mathrm{N}$ and $\mathrm{V}$, respectively, containing information of the candidate nanomaterials. These matrices represented graphically using line graph by plotting the magnitude of the attributes on the vertical axis and the attributes on the horizontal axis. Please note that the attributes, of which minimum values are preferred. If the values are plot for different candidate nanomaterials, obtain the line graph for them. These graphs distinct for all of the candidate nanomaterials and used as comparison basis. The area under the curve used to quantification purpose and to compare the candidate nanomaterials with each other and benchmark nanomaterials to be defined later.

Figure-3 line graph plot for the specifications and the area under the curve is shaded.

These line graphs plotted for specifications, normalized and weighted normalized specifications of all the candidate nanomaterials as well as the benchmark nanomaterials. The area under the curve obtained as follows. Let the width between the two parameters on horizontal axis as unity and $\mathrm{d}_{\mathrm{ij}}, \mathrm{n}_{\mathrm{ij}}$, and $\mathrm{v}_{\mathrm{ij}}$ are the elements of $\mathrm{D}, \mathrm{N}$, and $\mathrm{V}$ matrices.

Area under the line graph of specification of ith nanomaterial found out as:-

$$
\mathrm{AD}_{i}^{L}=\left(d_{i, 1}+2\left(d_{i, 2}+\cdots+d_{i, n-1}\right)+d_{i, n}\right) / 2
$$

Similarly, area under the graph of normalized and weighted normalized specifications of the ith nanomaterial, i.e. $\mathrm{AN}_{\mathrm{i}}^{\mathrm{L}}$ and $\mathrm{AV}_{\mathrm{i}}^{\mathrm{L}}$ using their respective elements.

\subsubsection{Identification and graphical representation of the benchmark nanomaterial.}

The same +ve benchmark nanomaterial, defined earlier, is used here for the comparison of the candidate nanomaterials for the ranking purpose. The areas under the line graph for +ve benchmark nanomaterial, i.e., $\mathrm{AD}^{\mathrm{L}}{ }_{\mathrm{B}}, \mathrm{AN}^{\mathrm{L}} \mathrm{B}$, and $\mathrm{AV}_{\mathrm{B}}^{\mathrm{L}}$ are calculated. All the candidate nanomaterials compared with the +ve benchmark nanomaterial for the evaluation purpose. It shows the suitability of the nanomaterials for the particular task.

\subsubsection{Ranking and selection of the nanomaterial.}

Now specification matrix is there along with normalized specification and weighted specification matrices ready for all the candidate nanomaterials along with the +ve benchmark nanomaterial. There is a need to measure and to compare the candidate nanomaterials with benchmark nanomaterial so that be ranked and selected.

\subsubsection{Coefficient of similarity (COS).}

The evaluation and ranking of the nanomaterials using the novel graphical methods done by their similarity to +ve benchmark nanomaterial. Let the Coefficient of similarity (COS) is the ratio of area under the curve or enclosed by the polygon for the candidate to that of the benchmark nanomaterial. The value of COS be any +ve fraction $(0 \leq \mathrm{COS} \leq 1)$ and a measure of the closeness of candidate nanomaterials with the benchmark nanomaterial. The candidates 
with COS magnitude closer to unity are preferable, since it indicates the closeness to the +ve benchmark nanomaterial.

According to TOPSIS method.

$\operatorname{COS}=\left(\mathrm{C}_{\mathrm{i}}^{*}-0\right), \operatorname{COS}^{\mathrm{D}}=\left(1-\mathrm{C}_{\mathrm{i}}^{*}\right)$, and $\operatorname{COS}+\operatorname{COS}^{\mathrm{D}}=\left(\mathrm{C}_{\mathrm{i}}^{*}-0\right)+\left(1-\mathrm{C}_{\mathrm{i}}^{*}\right)=1$.

Coefficient of similarity (COS) based on decision matrix

$\operatorname{COS}_{\mathrm{J}}^{\mathrm{D}}=\mathrm{AD}_{\mathrm{J}} / \mathrm{AD}_{\mathrm{I}}$

$\mathrm{AD}_{\mathrm{j}}$ for $\mathrm{jth}$ nanomaterial and different methods, i.e., line graph, etc.

Coefficient of similarity (COS) based on normalized specifications matrix

$$
\cos _{j}^{*}-\Lambda N_{j} / A N_{i}
$$

$\mathrm{AN}_{\mathrm{j}}$ for jth nanomaterial and different methods, i.e., line graph, etc.

Coefficient of similarity (COS) based on weighted normalized matrix

$$
\cos _{i}^{1}-\Lambda \mathrm{v}_{i} / \mathrm{AV}
$$

$\mathrm{AV}_{\mathrm{j}}$ for jth nanomaterial and different methods, i.e., line graph, etc.

Thus the COS calculations for all the $\mathrm{n}$ number of candidate nanomaterials are done by graphical methods, viz., line graph methods using the weighted normalized specifications.

\section{Illustrative example of ranking and optimum selection of nanomaterial}

Scientists, engineers and product developers may use the following example for implementation of proposed methodology.

\section{Stage-1.Elimination Search Method}

Identify the application and corresponding pertinent attributes. Also define the requirements of research and product development carefully. Because without identify the applications, identifying the pertinent attributes, defining the requirements he will never eliminate the large list of nanomaterial to a manageable list. In this example, "manufacturing of industrial nanodevice" is taken as application. For this, minimum requirements of the nanomaterials that are required summarized in Table-10.

The minimum requirements for application considered as shown in Table $\mathbf{1 0 .}$

After defining the requirements applied the pertinent attributes, requirements and eliminate infeasible nanomaterials (elimination done on the basis of pertinent attributes one by one) from the available ' $n$ ' attributes database/knowledge base and prepare a manageable list of nanomaterials.

Here, in this example 31 standard nanomaterials with their pertinent attributes have been taken for optimum selection of nanomaterials as per the given application.

List of 31 nanomaterials are shown in Table-9 
Out of these 31 standard nanomaterials only 7 nanomaterials with their pertinent attributes are best suited for the given application for the selection of best/optimum nanomaterials and remaining 24 standard nanomaterials are eliminated due to the insufficient significance of their attributes as per the given application.

Now a manageable list of 7 standard nanomaterials with five pertinent attributes is formed which best suits as per the given application are shown in Table 11.

\section{Stage-2.Evaluation using TOPSIS method}

\section{Step-1.Formation of decision matrix, ' $D$ '}

By using Table 11 Prepare a decision matrix. The matrix which will contain all the magnitudes of specifications. In this matrix, rows represent the candidate nanomaterials and the column represents the pertinent attributes.

In this example 7 candidate nanomaterials and 5 attributes are considered with their values are listed below in Equation (16).

$$
\mathrm{D}=\left[\begin{array}{ccccc}
193 & 79 & 2850 & 12 & 205 \\
212 & 910 & 1870 & 15 & 71 \\
160 & 1020 & 2520 & 26 & 1000 \\
189 & 411 & 2730 & 62 & 58 \\
280 & 60 & 2509 & 50 & 70 \\
256 & 168 & 1705 & 34 & 150 \\
290 & 107 & 3000 & 55 & 1000
\end{array}\right]
$$

Attributes for the short listed candidate nanomaterials as per the given application are listed in Table 11

\section{Step-2. calculating the normalized specification matrix using Esq.-(1)}

The normalization helps to provide the dimensionless elements of the matrix.

$$
\mathrm{n}_{\mathrm{ij}} \quad=d_{i j} /\left(\sum_{i=1}^{m} d_{i j}^{2}\right)^{1 / 2}
$$




$\mathrm{N}=\left[\begin{array}{lllll}0.3166 & 0.0546 & 0.4314 & 0.1115 & 0.1422 \\ 0.3477 & 0.6210 & 0.2831 & 0.1394 & 0.0492 \\ 0.2624 & 0.7060 & 0.3815 & 0.2417 & 0.6937 \\ 0.3100 & 0.2845 & 0.4133 & 0.5764 & 0.0402 \\ 0.4593 & 0.0415 & 0.3798 & 0.4648 & 0.0485 \\ 0.4110 & 0.1162 & 0.2581 & 0.3160 & 0.1040 \\ 0.4757 & 0.0740 & 0.4542 & 0.5113 & 0.6937\end{array}\right] \ldots \ldots$.

\section{Step 3.Construction of relative importance matrix A.}

To find out the weight vector for different attributes of nanomaterials for given application, the relative importance matrix is developed.

A questionnaire form is prepared explaining the requirements of the application, different scientists, product developers and experts are asked to fill up these forms independently and then average is taken to find out the values of all the off-diagonal elements.

The values in the lower diagonal matrix cells are reciprocal of the corresponding values in the cells of upper diagonal matrix. Alternatively, this process may be completed by a team of experts/stakeholders.

Only pair-wise comparision is permitted in this method. i.e. $a_{\mathrm{ij}}=\mathrm{w}_{\mathrm{i}} / \mathrm{w}_{\mathrm{j}}$, where this ratio represents the relative importance of $\mathrm{i}^{\text {th }}$ attribute with respect to the $\mathrm{j}^{\mathrm{t} \text { th }}$ attribute corresponding to the given application. Relative importance matrix for given application is shown below:

$\mathrm{A}=\left[\begin{array}{ccccc}1 & 1 & 2 & 0.5 & 0.33 \\ 1 & 1 & 0.5 & 2 & 2 \\ 0.5 & 2 & 1 & 3 & 2 \\ 2 & 0.5 & 0.33 & 1 & 0.33 \\ 3 & 0.5 & 0.5 & 3 & 1\end{array}\right]$

\section{Step-4.Find out the maximum Eigen value of the relative importance matrix $A$}

Eigen value formulation provides to find out the weight vector as shown below:- 
$(\mathrm{A}-\lambda \mathrm{I}) \mathrm{W}=0$

Where, I is the identity matrix, and $\mathrm{W}$ is the weight vector.

This equation can also be written as

$(\mathrm{A}-\lambda \mathrm{I})=0$ and $\mathrm{W}=0$, Where this gives a trivial solution having no meaning.

$(\mathrm{A}-\lambda \mathrm{I})=\left[\begin{array}{ccccc}1-\lambda & 1 & 2 & 0.5 & 0.33 \\ 1 & 1-\lambda & 0.5 & 2 & 2 \\ 0.5 & 2 & 1-\lambda & 3 & 2=0 \\ 2 & 0.5 & 0.33 & 1-\lambda & 0.33 \\ 3 & 0.5 & 0.5 & 3 & 1-\lambda\end{array}\right]$

Characteristics Polynomial Equation

$-\lambda^{5}+5 \lambda^{4}-0.03 \lambda^{3}+25.85 \lambda^{2}+21.88 \lambda+9.767=0$

After solving the characteristics polynomial equation, by using the MATLAB $\lambda_{\max }$ is calculated. By solving we get 5 values of $\lambda$ as shown below.

$\lambda=6,-0.2095 \pm 2.2910 i,-0.2762 \pm 0.4806 i$

From this, $\lambda=6$ is the maximum value, as by taking the largest $\lambda$ value the correct equation is obtained.

Therefore, $\lambda_{\max }=\mathbf{6}$. Now put this value in Equation (21)

$\left(\mathrm{A}-\lambda_{\max } \mathrm{I}\right)=\left[\begin{array}{ccccc}-5 & 1 & 2 & 0.5 & 0.33 \\ 1 & -5 & 0.5 & 2 & 2 \\ 0.5 & 2 & -5 & 3 & 2 \\ 2 & 0.5 & 0.33 & -5 & 0.33 \\ 3 & 0.5 & 0.5 & 3 & -5\end{array}\right]$

Step-5.Calculating weights for each attribute using the Eigen vector associated with maximum Eigen value. 


$$
\left(\mathrm{A}-\lambda_{\max } \mathrm{I}\right) \mathrm{W}=0
$$

This Equation (23) will give a set of linear equations on the basis of this it is calculated.

$\left(\mathrm{A}-\lambda_{\max } \mathrm{I}\right) \mathrm{W}=\left[\begin{array}{ccccc}-5 & 1 & 2 & 0.5 & 0.33 \\ 1 & -5 & 0.5 & 2 & 2 \\ 0.5 & 2 & -5 & 3 & 2 \\ 2 & 0.5 & 0.33 & -5 & 0.33 \\ 3 & 0.5 & 0.5 & 3 & -5\end{array}\right]\left[\begin{array}{l}\mathrm{W} 1 \\ \mathrm{~W} 2 \\ \mathrm{~W} 3 \\ \mathrm{~W} 4 \\ \mathrm{~W} 5\end{array}\right]$

Summation of all the weight vectors is equal to unity.

$$
w_{1}+w_{2}+w_{3}+w_{4}+w_{5}=1
$$

$\mathrm{W} 1=0.1761, \mathrm{~W} 2=0.2042 . \mathrm{W} 3=0.2668, \mathrm{~W} 4=0.2430, \mathrm{~W} 5=0.2286$

Step-6.Calculating the weighted normalized specification matrix using Esq.-(8).

Here incorporate the relative importance of the attributes with their normalized value to create unique parameter for the candidate nanomaterial

$V=\left[\begin{array}{lllll}0.0557 & 0.0111 & 0.1150 & 0.0270 & 0.0325 \\ 0.0612 & 0.1268 & 0.0755 & 0.0338 & 0.0112 \\ 0.0462 & 0.1441 & 0.1017 & 0.0587 & 0.1585 \\ 0.0545 & 0.0580 & 0.1102 & 0.1400 & 0.0091 \\ 0.0808 & 0.0084 & 0.1013 & 0.1129 & 0.0110 \\ 0.0723 & 0.0237 & 0.0688 & 0.0767 & 0.0237 \\ 0.0837 & 0.0151 & 0.1211 & 0.1242 & 0.1585\end{array}\right] \ldots \ldots \ldots$


The weighted normalized specification matrix is all-inclusive matrix, which takes care of the attribute values and their relative importance. So the matrix will be able to provide good basis for comparison with each other and with the benchmark nanomaterials.

\section{Step-7.Generation of +ve and -ve benchmark nanomaterials and separation measures}

The weighted normalized attributes for the +ve and _ve benchmark nanomaterials be obtained as:-

The weighted normalized attributes for the +ve benchmark nanomaterials is calculated by taking the largest value from all the columns with respect to all the pertinent attributes in correspondence to their candidate nanomaterials.

Theoretically best solution of nanomaterials

$\mathrm{V}^{*}=(0.0837,0.1441,0.1211,0.1400$, and 0.1585$)$

Similarly, The weighted normalized attributes for the -ve benchmark nanomaterials is calculated by taking the smallest value from all the columns with respect to all the pertinent attributes in correspondence to their candidate nanomaterials.

Theoretically worst solution of nanomaterials

$\mathrm{v}^{-}=(0.0462,0.0084,0.0688,0.0270$, and 0.0091$)$

Values of Separation from the +ve benchmark nanomaterials $\mathbf{S}^{*}$ and Values of Separation from the -ve benchmark nanomaterials $\mathbf{S}^{-}$is given below in Table 12.

\section{Step-8.Suitability Index}

Then the relative closeness to the +ve benchmark nanomaterials, $C^{*}$, which is a measure of the suitability of the nanomaterials for the chosen application on the basis of attributes considered, is calculated. A nanomaterial with the largest $C^{*}$ is preferable.

$\mathrm{C}_{\mathrm{i}}^{*}=\mathrm{S}_{\mathrm{i}}^{-} / \mathrm{S}_{\mathrm{i}}^{*}+\mathrm{S}_{\mathrm{i}}^{-} \quad$ Where $\mathrm{i}=1, \ldots . ., \mathrm{m}$.

Ranking of the candidate nanomaterials is done in accordance with the decreasing values of indices $\mathrm{C}_{\mathrm{i}}$ * indicating the most preferred and the least preferred feasible optional solutions. This index may be called as suitability/goodness index.

Relative closeness to ideal solution

$\mathrm{C}^{*}=0.1953$
$\mathrm{C}^{*}=0.3873$
$\mathrm{C}^{*}{ }_{3}=0.6931$
$\mathrm{C}_{4}=0.4267$
$\mathrm{C}^{*}{ }_{5}=0.3255$
$\mathrm{C}^{*}=0.2041$
$\mathrm{C}^{*}=0.5934$ 
Evaluation and ranking of the candidate nanomaterials using TOPSIS methods as shown in Table 13.

\section{Computer Program MATLAB}

A MATLAB program is developed for performing calculations of above procedure from steps 1-8. The MATLAB program developed requires decision matrix ' $D$ ' and relative importance matrix ' $A$ ' as input and after performing the remaining calculation gives ranking in the form of $\mathrm{C}^{*}$ i as output.

The selection procedure described in section 4 is iterated for each candidate nanomaterials to arrive at optimum nanomaterials for application under consideration.

\section{Graphical method based Ranking}

The element values of weighted normalized specification matrix are used for the line graph plotting. Subsequently, COS can be calculated from graphs. The calculated COS is tabulated as follows.

Suppose the area under the line graph for weighted normalized specifications of first candidate nanomaterial and for benchmark nanomaterial are $\mathrm{AV}_{1}{ }^{\mathrm{L}}=0.1972 ; \mathrm{AV}_{+\mathrm{B}}{ }^{\mathrm{L}}=0.5263$. The coefficient of similarity based on the weighted normalized specification of the first candidate nanomaterial is

$\mathrm{COS}_{1}{ }^{\mathrm{VL}}=\mathrm{AV}_{1}^{\mathrm{L}} / \mathrm{AV}_{+\mathrm{B}}^{\mathrm{L}}=0.3746$

Similarly closeness of the candidate nanomaterials with the +ve benchmark nanomaterials obtained from TOPSIS and the graphical methods are tabulated as shown in Table 13 Thus the nanomaterials are ranked in order of preference based on the attributes selected. For the purchase of a new nanomaterials, the management can use the above ranking effectively to select the nanomaterials, which will be best suitable for the application and is based on this set together with other considerations.

\section{Role of user in selection}

Here one see, the ranking done by TOPSIS and graphical methods vary from each other. Even for both graphical methods the ranking is not same. The user find out which method is the best suited for his application. Thus the nanomaterials are ranked in order of preference based on the attributes are selected. However, before a final decision is taken to purchase a new nanomaterial, the following factors come into picture: (1) Economic considerations, (2) Availability, (3) Management constraints, corporate policies, (5) SWOT analysis, (by Keeping the short term and long term objectives in mind, comprehensive SWOT analysis by the designer, device manufacturer and R \& D organizations helps in the development of creative and innovative nanodevices) (4) International market policies, which were not previously considered in coding and evaluation. Even if the above consideration, say, economic considerations, does not allow the user to buy the top ranked nanomaterials, the user knows which one to go for as the next choice. For example, 2nd and 3rd ranked nanomaterial costing the same, but as our result indicates, the 2 nd ranked nanomaterial will perform better in other aspects even though their price is same. 


\section{Step-by-step procedure for optimum selection of nanomaterial}

Step-1:- Decide about the aims and objective for which nanomaterials is to be used.

Step-2:-Identify all the possible alternative nanomaterials available in the literature.

Step-3:-Use cause and effect diagram to find out different classes/groups of attributes/properties/characteristics and different attributes in identified classes.

Step-4:-Develop an n-digit coding scheme for characterization/specification for storage and retrieval in the computer.

Step-5:-Carry out elimination search to reduce the large list of alternatives nanomaterials as $1^{\text {st }}$ stage of $3^{\text {rd }}$ stage selection procedure.

Step-6:-Select TOPSIS (Technique for Order Preferences by Similarity to Ideal Solution) as attribute based evaluation procedure for this small list of alternatives.

Step-7:-After evaluation, rank the nanomaterials in order of preference for given application.

\section{Results and Discussion}

Results: Here, illustrative problem of ranking and selection of nanomaterials is solved using TOPSIS method, which is described in Section 4 and according to step $\mathrm{V}$ of proposed method, normalized value of material selection attributes for nanomaterials are calculated by using Equation (17) .The normalized data of the nanomaterial selection attributes are shown in Normalized specification matrix as Equation (18) ) and also by graphical method. The problem considering seven candidate nanomaterials and five attributes as shown in Table 11. In this example, specific surface area of nanomaterial is non-beneficial attributes and the remaining 4 attributes are beneficial attributes. By applying the 3-stage procedure for optimum selection and ranking of nanomaterials as discussed in section 4 of proposed methodology, results are obtained. The results for the selection and ranking of nanomaterials are obtained via, TOPSIS method and Graphical methods and it is compared with each other for selection of nanomaterials for given application as given in Table 13.The evaluation and ranking of the nanomaterials using the novel graphical methods done by their similarity to +ve benchmark nanomaterials

Discussion: Attribute based characterization helps the scientists and product developers working in the area of nanotechnology to understand different nanomaterials in depth for possible R \& D applications and developing new nanoproducts. The attribute knowledgebase grows with the addition of new nanomaterials and new attributes for present and future applications.

The effect of units and magnitudes of the attributes is normalized, Aims and objectives of $\mathrm{R} \& \mathrm{D}$ in the development of new methodology and nanoproducts decide the relative importance of attributes which is suitably addressed in the methodology.

* It is absolutely necessary that all the attribute information must be available in the attribute knowledgebase. Special efforts must be made in this direction.

* MATLAB software can be used to find out the weights for different attributes for given application and suitability index $\mathrm{C}_{\mathrm{i}}$ * for ranking purpose. 
The method is flexible enough and permits selection of optimum nanomaterials in the presence of large number of feasible materials and attributes. The method ensures that the selected (optimum) nanomaterial is closest to the hypothetical best nanomaterial and farthest from hypothetical worst nanomaterial.

* The methodology is illustrated in this paper for optimum selection of nanomaterials. Work is in progress to select optimally different subsystems in an integrated way in the overall nanotechnology project.

Here, TOPSIS method is used and obtained the ranking an order of ideal solution is $\mathrm{N}_{3}>\mathrm{N}_{7}>$ $\mathrm{N}_{4}>\mathrm{N}_{2}>\mathrm{N}_{5}>\mathrm{N}_{6}>\mathrm{N}_{1}$, whereas graphical method suggests the ranking an order of ideal solution is $\mathrm{N}_{7}>\mathrm{N}_{3}>\mathrm{N}_{4}>\mathrm{N}_{5}>\mathrm{N}_{2}>\mathrm{N}_{6}>\mathrm{N}_{1}$. It is recommend that nanomaterial alternative $\mathrm{N}_{3}$, i.e. QGraphene ${ }^{\circledR}-50$ is the first choice, and Q Tubes ${ }^{\circledR} 250$, QSI-Nano ${ }^{\circledR}$ Silver, NanoXactTM AU Nanoparticle, AgNW60, NanoPore ${ }^{\mathrm{TM}} \mathrm{HP}-150$, and AgNW115 are placed in the second, third, fourth, fifth, sixth and seventh choices, respectively. Whereas, Graphical method suggests nanomaterial alternative $\mathrm{N}_{7}$, i.e. Q Tubes ${ }^{\circledR} 250$ is the first choice, QGraphene ${ }^{\circledR}-50$, QSI-Nano® Silver, are second and third choice, , AgNW60, NanoXactTM AU Nanoparticle are placed in fourth and fifth choice and NanoPore ${ }^{\mathrm{TM}}$ HP-150 and AgNW115 placed in sixth and seventh place respectively. Both the methods suggest that material alternative $\mathrm{N}_{1}$, i.e. AgNW115 is the last choice. According to the TOPSIS method QGraphene ${ }^{\circledR}$ - 50 is the best alternative because of high thermal conductivity, young's modulus and aspect ratio this alternative is selected as most appropriate for the considered application.

\section{Conclusions}

The paper presents a nanomaterial selection procedure based on the Multiple Attribute Decision Making (MADM) approach, which is a concept used not so frequently for the purpose. It identifies the various attributes needing to be considered for the optimum evaluation and selection of nanomaterial. It provides a coding system for nanomaterials depicting the various attributes. It recognizes the need for, and processes the information about, relative importance of attributes for a given application without which inter-attribute comparison is not possible. It presents the result of the information processing in terms of a merit value, which is used to rank the nanomaterials in the order of their suitability for the given application. The contributions of the work be summarized as

1. The method is especially suitable for generating data of nanomaterials available in the market and their subsequent retrieval. It provides coding scheme to produce electronic database of globally available nanomaterials.

2. The data will be helpful to all sorts of people related to nanomaterials from manufacturer, designers, and users to maintenance personnel. It will be helpful to improve the overall productivity of the organization.

3. Here by identifying 168 attributes of the nanomaterials, the attempt has been made to codify most of the nanomaterial characteristics, which will define the nanomaterial precisely and accurately. The coding scheme is illustrated with example.

4. Evaluation and ranking based on the mathematical and graphical approaches along with the illustrative examples are given.

5. MATLAB program is developed for performing calculations involved.

\section{References}


[1] Fisher PE, Lawrence W. Selection of engineering materials and adhesives. Taylor \& Francis Group: CRC Press; 2005.

[2] Ashby MF, Johnson K. Materials and design: the art and science of materials selection in product design. Oxford: Butterworth Heinemann; 2002.

[3] Farag M. Materials selection for engineering design. New York: Prentice-Hall; 1997.

[4] Edwards KL. Selecting materials for optimum use in engineering components. Mater Des 2005; 26:469-73.

[5] Deng YM, Edwards KL. The role of materials identification and selection in engineering design. Mater Des 2007; 28:131-9.

[6] Edwards KL, Deng YM. Supporting design decision-making when applying materials in combination. Mater Des 2007; 28:1288-97.

[7] Rao RV, Davim JP. A decision-making framework model for material selection using a combined multiple attributes decision-making method. Int J Adv Manuf Technol 2008; 35:751-60.

[8] Shanian A, Savadogo O. A material selection model based on the concept of multiple attribute decision-making. Mater Des 2006; 27:329-37.

[9] Shanian A, Savadogo O. A non-compensatory compromised solution for material selection of bipolar plates for polymer electrolyte membrane fuel cell (PEMFC) using ELECTRE IV. Electrochim Acta 2006; 51:5307-15.

[10] Shanian A, Savadogo O. TOPSIS multiple-criteria decision support analysis for material selection of metallic bipolar plates for polymer electrolyte fuel cell. J Power Sources 2006; 159:1095-104.

[11] Rao RV. A decision-making methodology for material selection using an improved compromise ranking method. Mater Des 2008; 29:1949-54.

[12] Wang MJJ, Chang TC. Tool steel materials selection under fuzzy environment. Fuzzy Sets Syst 1995; 72:263-70.

[13] Liao TW. A fuzzy multi criteria decision-making method for material selection. J Manuf Syst 1996; 15:1-12.

[14] Chen SJ, Hwang CL. Fuzzy multiple attribute decision-making methods and applications. Lecture notes in economics and mathematical systems. Berlin: Springer-Verlag; 1992.

[15] Hwang CL, Yoon KP. Multiple attribute decision-making: methods and applications. New York: Springer; 1991.

[16] Rao RV. Decision making in the manufacturing environment using graph theory and fuzzy multiple attribute decision-making methods. London: Springer- Verlag; 2007.

[17] Roa RV. A material selection model using graph theory and matrix approach. Mater Sci Eng A 2006; 431:248-55.

[18] Manshadi BD, Mahmudi H, Abedian A, Mahmudi R. A novel method for materials selection in mechanical design: combination of non-linear normalization and a modified digital logic method. Mater Des 2007; 28:8-15.

[19] Chan JWK, Tong TKL. Multi-criteria material selections and end-of-life product strategy: grey relational analysis approach. Mater Des 2007; 28:1539-46.

[20] Chatterjee Prasenjit, Athawale Vijay Manikrao, Chakraborty Shankar. Selection of materials using compromise ranking and outranking methods. Mater Des 2009; 30(10):404353.

[21] Ashby MF. Multi-objective optimization in material design and selection. Mater Des 2000; 48:359-69.

[22] Khabbaz SR, Manshadi BD, Abedian A, Mahmudi R. A simplified fuzzy logic approach for materials selection in mechanical engineering design. Mater Des 2009; 30:687-97. 
[23] Fayazbakhsh K, Abedian A, Manshadi BD, Khabbaz SR. Introducing a novel method for materials selection in mechanical design using Z-transformation in statistics for normalization of material properties. Mater Des 2009; 30: 4396-404.

[24] Saaty TL. Fundamentals of decision-making and priority theory with the AHP. Pittsburg: RWS Publications; 2001.

[25] Venkatasamy R, Agrawal VP. A digraph approach to quality evaluation of an automotive vehicle. Qual Eng 1997; 9:405-17.

[26] Suresh Babu K, Subba Raju NV, Srinivas Reddy M, Nageswara Rao D. The material selection for typical wind turbine blades using a MADM approach \& analysis of blades. In: Proceeding of MCDM, Chania, Greece: June 19-23; 2006.

[27] Zadeh L. Fuzzy Sets. Information and Control. 1965; pages 338-353.

[28] Bellman R. and Zadeh L. Decision Making in a Fuzzy Environment. Management Science.1970; 17B (4):141-164.

[29] Gau W. and Buehrer D. Vague Sets. IEEE Transactions on Systems, Man, and Cybernetics. 1993; 23:610-614.

[30] Opriumic S. and Tzeng G. Compromise solution by MCDM methods: A comparative analysis of VIKOR and TOPSIS. European Journal of Operational Research.2004; 156(2):445455.

[31] Chu M. and Shyu J. Comparison among three analytical methods for knowledge communities group-decision analysis. Expert Systems with Application.2007; 33(4):10111024.

[32] Opricovic S. Multi-criteria Optimization of Civil Engineering Systems. Technical report, Belgrade. 1998.

[33] Hwang C. and Yoon K. Multiple Attributes Decision Making Methods and Applications: a state-of-the-Art Survey. Springer, Berlin Heidelberg 1981.

[34] Chen C. Extension of the TOPSIS for Group Decision making under Fuzzy Environment. Fuzzy Sets and Systems.2000; 114:1-9.

[35] Silvio G. Interval TOPSIS for Multi-criteria Decision Making. Neural Nets.2002; 89(2):56-63.

[36] Serafim O. and Tzeng G. Compromise Solution by MCDM Methods: A Comparative Analysis of VIKOR and TOPSIS. European Journal of Operational Research.2004; 156:445455.

[37] C.L. Hwang, M.J. Lin, Group decision making under multiple criteria, methods and applications, Lecture notes in Economics and mathematical systems, Springer-Verlag, Berlin, Heidelberg, 1987.

[38] G. Strang, Linear Algebra and Its Applications, Harcourt Brace Jovanovich, Publishers, 1980.

\section{Figure Captions}

Figure-1 cause and effect analysis of nanomaterial characterization.

Figure-2 swot analysis for manufacturer.

Figure-3 line graph plot for the specifications and the area under the curve is shaded. 